\title{
Ultimate strength of a tungsten heavy alloy after severe plastic deformation at quasi-static and dynamic loading
}

\author{
Lothar W. Meyer ${ }^{1,}$, Matthias Hockauf ${ }^{1, b}$, Anton Hohenwarter ${ }^{2, ~ c}$, Steffen \\ Schneider ${ }^{3, d}$
}

${ }^{1}$ Chemnitz University of Technology, Department of Materials and Impact Engineering, Erfenschlager Str. 73, 09125 Chemnitz, Germany

\author{
${ }^{2}$ Erich Schmid Institute of Material Sciences, CD-Laboratory for Local Analysis of Deformation and \\ Fracture, Austrian Academy of Sciences, Jahnstr. 12, A-8700 Leoben, Austria \\ ${ }^{3}$ Wehrwissenschaftliches Institut für Werk-, Explosiv- u. Betriebsstoffe (WIWEB), Erding, Germany \\ alothar.meyer@mb.tu-chemnitz.de, ${ }^{b}$ matthias.hockauf@mb.tu-chemnitz.de, \\ canton.hohenwarter@unileoben.ac.at, ${ }^{d}$ steffenschneider@bwb.org
}

\begin{abstract}
Keywords: WHA, High-pressure torsion, Strain hardening, Strain rate sensitivity, Ultrafine-grained microstructure, Saturation, Shearband, Nanocrystalline
\end{abstract}

\begin{abstract}
A tungsten heavy alloy ( $92 \% \mathrm{~W}, \mathrm{Ni}-\mathrm{Co}$ matrix) is subjected to severe plastic deformation (SPD) by high pressure torsion (HPT) at room temperature up to equivalent strains of $0.7,5.3,10.7$ and 14.3. The microstructure and the mechanical properties are investigated by cylindrical compression samples at quasi-static and dynamic loading. The harder spherical $\mathrm{W}$ particles are homogeneously deformed within the softer matrix, becoming ellipsoidal at medium strains and banded at high strains without shear localization or fracture. Results of quasi-static loading show that the strength is approaching a limiting value at strains of $\sim 10$. At this strain for the matrix a grain size of $\sim 80 \mathrm{~nm}$ and for $\mathrm{W}$ a cell size of $\sim 250 \mathrm{~nm}$ was observed, suggesting strain concentration on the matrix. The initial yield stress of $945 \mathrm{MPa}$ for the coarse-grained condition is increased thereby to an ultimate value of $3500 \mathrm{MPa}$, while a peak stress of $\sim 3600 \mathrm{MPa}$ is reached. Such remarkably strength has never been reported before for pure $\mathrm{W}$ or $\mathrm{W}$-based composites. The strain hardening capacity as well as the strain rate sensitivity is reduced drastically, promoting the early formation of (adiabatic) shear bands.
\end{abstract}

\section{Introduction}

The most common tungsten heavy alloys (WHAs) are types of sintered multiphase metallic composites, consisting of tungsten particles (bcc-structure) in a comparatively ductile nickel, iron or copper binder matrix (fcc-structure) [1]. In conventional heavy alloys, the tungsten content ranges from 90 to 98 weight pct. Because of their unique combination of high strength and ductility, high modulus, corrosion resistance and density they are used for a number of applications including radiation shields, counterweights, electrical contacts, armor, and kinetic energy penetrators.

Due to their unique properties, bulk metals with submicrocrystalline or ultrafine-grained microstructure processed by methods of severe plastic deformation (SPD) have attracted the increasing interest of many researchers during the last decades [2]. It is well known that conventional plastic deformation results in a refinement of the microstructure where dislocations are heterogeneously distributed, forming a cellular structure with low misorientations. In contrast SPDprocessed materials possess a granular structure containing a significant number of high angle grain boundaries [2, 3]. Due to recent improvements in tool design, special technical solutions were evolved, which enabled the processing of difficult-to-work materials and the "scaling up" for a significant increase in production efficiency $[2,4]$. Recent papers report the application of SPD on titanium alloys [5], low and medium carbon steels [6-8], tungsten composites [9] or even pure tungsten [10-13]. Within a various number of different SPD-techniques [2] the most utilized are Equal Channel Angular Extrusion (ECAE) and High Pressure Torsion (HPT). The former because 
of the possibility for producing billets big enough for practical applications and the latter because of the extreme strains which can be reached at high pressures and low temperatures even for difficultto-work materials. The present paper deals with the development of microstructure and mechanical properties of a commercial WHA processed by HPT at room temperature (RT). So far, data for SPD-processed WHA is missing in the literature.

\section{Experimental}

The investigated material is a conventional, sintered W-Ni-Co tungsten heavy alloy (92 wt. pct. W). For HPT-processing the disc-shaped material (diameter $\sim 8 \mathrm{~mm}$, height $\sim 0.8 \mathrm{~mm}$ ) was processed between two anvils with a cylindrical cavity. By the rotation of one of the anvils a simple shear deformation was generated due to the contact friction at the end faces. An axial nominal pressure of 7.8 GPa was applied to avoid sliding. All samples were marked with a line on both end faces before deformation. If the lines were visible after deformation, a slip-free deformation was verified. The HPT-experiments were carried out at RT with a rotation speed of 0.2 revolutions per minute. With reference to a radius of $3 \mathrm{~mm}$ the discs were strained up to an equivalent strain $\varepsilon_{\mathrm{HPT}}$ of $0.7,5.3,10.7$ and 14.3. The applied strains roughly correspond to that induced by 1, 8, 16 and 20 ECA-extrusions in a $120^{\circ}$-die. Further details about the used HPT facility are presented in [3].

For the investigation of flow stress as a function of strain and strain rate, cylindrical miniature compression samples (diameter $1 \mathrm{~mm}$, height $0.7 \mathrm{~mm}$ ) were extracted from the discs by micromachining. According to Figure 1a-c eight samples per disc were taken along the axial direction at a radius of $3 \mathrm{~mm}$. It is important to note that because of the radius-dependence of the deformation there is a strain-gradient across the discs as well as across the compression samples. As shown by [14] the gradient gets less remarkable with increasing strain, especially for the considered radius of $3 \mathrm{~mm}$. Nevertheless it should be kept in mind for the later interpretation of the mechanical response, especially for the yielding.

Quasi-static compression tests at initial strain rates of $3 \cdot 10^{-4} \mathrm{~s}^{-1}$ were operated in a conventional testing machine from Zwick-Roell. Force measurement was done by the adjusted load cell. Inductive transducers, mounted to a small load frame designed for testing of miniature compression samples, were used for strain measurement. Dynamic compression tests at initial strain rates of $6 \cdot 10^{2} \mathrm{~s}^{-1}$ were realized by a small drop-weight facility with a guided mass of $3.4 \mathrm{~kg}$. The force was calculated according to Hookes Law from the elastic deformation of the punch measured by permanent strain gauges. The strain was measured by a non-contacting electro-optical high-speed extensometer from Zimmer following two check marks above and below the sample. This setup enables the measurement of high quality stress-strain data with insignificant inertia effects at high strain rates.
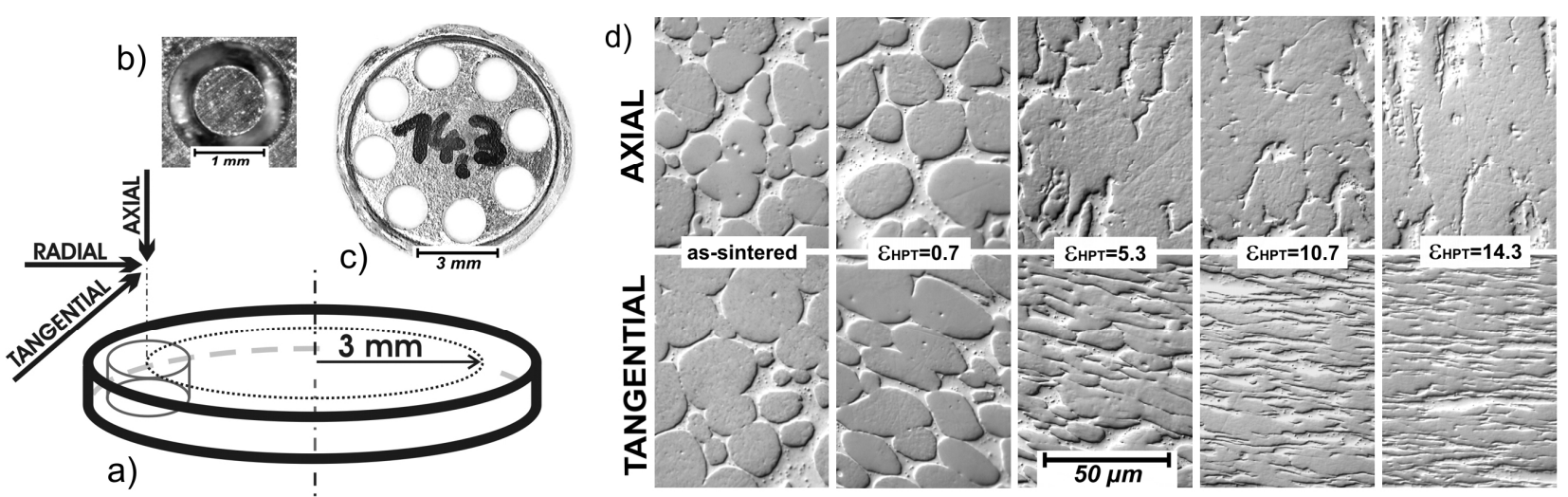

Fig. 1: Details for the investigated material. a) Schematic of a HPT-disc with definition of the investigated directions, b) Micromachined compression sample before final extraction, c) Asprocessed HPT-disc $\left(\varepsilon_{\mathrm{HPT}}=14.3\right)$ after extraction of the compression samples, d) Microstructure of the investigated material before and after HPT-processing (same scale for all micrographs). 
For the dynamic as well as for the static compression tests yttrium-stabilized zirconium adaptorplates were used to avoid penetration of the punches due to the high strength of the material. The front faces of the specimens were lubricated with high performance $\mathrm{MoS}_{2}$ grease. At least three parallel tests were performed for each condition.

Investigations of the microstructure were performed at a radius of $3 \mathrm{~mm}$ of the discs by optical microscopy and transmission electron microscopy (TEM) in a Jeol-2010 from HITACHI operated at $200 \mathrm{kV}$. For TEM-investigations the HPT-discs were mechanically thinned to $\sim 60 \mu \mathrm{m}$. Since punching was not possible, the samples for TEM-investigations were taken by a supersonic driven core drill. Further thinning was performed by ion etching in Ar-atmosphere with an angle of $\sim 5^{\circ}$ at $4 \mathrm{keV}$ until perforation.

\section{Results and discussion}

There are two reports of Wei et al. dealing with the microstructure and the mechanical properties of SPD-processed pure $\mathrm{W}$ at quasi-static and dynamic loading. Therein the material was either ECAEprocessed slightly below recrystallisation temperature at $1000^{\circ} \mathrm{C}$ followed by rolling at temperatures $>600^{\circ} \mathrm{C}$ [12] or HPT-processed at $500^{\circ} \mathrm{C}$ [13]. It was shown that the HPT-processing at lower temperature was more effective for achieving small grains and remarkably high strengths.

Figure 1d illustrates the development of the microstructure of the HPT-processed WHA in the axial and tangential section. The spherical tungsten particles of the as-sintered condition have an average diameter of $\sim 20 \mu \mathrm{m}$. They are homogeneously distributed within the Ni-Co matrix. In the tangential section the particles become ellipsoidal with increasing shear strain and later form a banded structure. Accordingly they show a plate-like appearance in the axial section. In contrast to [9] neither broken tungsten particles nor strain localisations were found. This might be attributed to the different chemical composition of the matrix as well as the different $\mathrm{W}$-content.

Figure 2a displays a bright-field TEM micrograph showing the microstructure of the as-sintered condition. Inside the $\mathrm{W}$-particles a uniform structure of individual dislocations is visible. The selected area electron diffraction patterns (SAED) of both the Ni-Co matrix and the W-particles show concentrated intensities, revealing a single crystalline structure with identical orientation. Micrographs after $\varepsilon_{\mathrm{HPT}}=0.7$ and $\varepsilon_{\mathrm{HPT}}=5.3$ (not shown) revealed a cellular substructure in both the matrix and the $\mathrm{W}$. Figure $2 \mathrm{~b}$ shows a dark-field micrograph of the Ni-Co matrix after a $\varepsilon_{\mathrm{HPT}}=10.7$. Rather equiaxed grains with an average size of $\sim 80 \mathrm{~nm}$ were generated at this stage of deformation. The smallest grains have a diameter of $\sim 30 \mathrm{~nm}$ that is well in the nanocrystalline range [2]. The grain boundaries are mostly of high-angle type, which is indicated by the clearness of their appearance and the diffraction beams forming almost continuous rings.
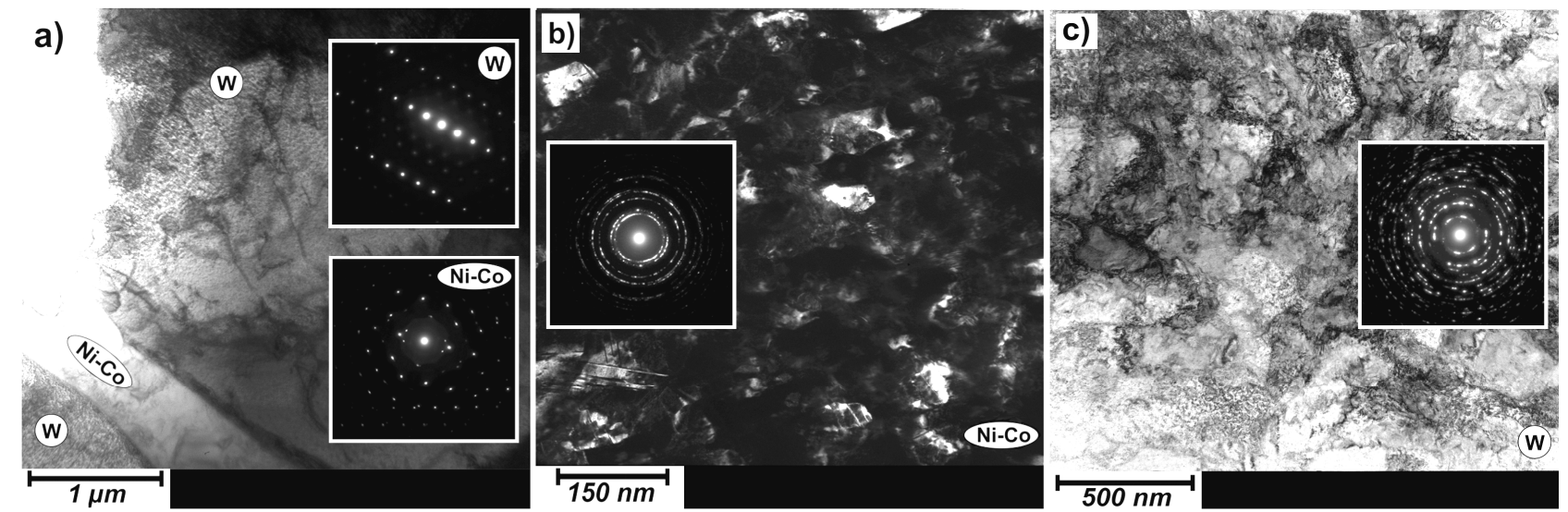

Fig. 2: TEM-micrographs with SAED of the a) as-sintered condition (bright-field), b) after $\varepsilon_{\mathrm{HPT}}=10.7$ for Ni-Co (dark-field) and c) after $\varepsilon_{\mathrm{HPT}}=10.7$ for $\mathrm{W}$ (bright-field). 
The corresponding TEM-micrograph for the $\mathrm{W}$ after $\varepsilon_{\mathrm{HPT}}=10.7$ is shown in Figure $2 \mathrm{c}$ where the boundaries are less well-defined inside the highly dislocated microstructure. The SAED shows concentrations suggesting the coexistence of low- and high-angle grain boundaries. The average cell size is $\sim 250 \mathrm{~nm}$. This suggests that the formation of truly ultrafine- or nanocrystalline grains for the $\mathrm{W}$ is not completed at this strain. In contrast, in the Ni-Co matrix such a microstructure forms above $\varepsilon_{\mathrm{HPT}}=5.3$. Consequently, the deformation seems to be increasingly carried by the softer matrix. This assumption is supported by the results of [13] where, despite the higher processing temperatures, a grain size of $\sim 150 \mathrm{~nm}$ was reported for pure $\mathrm{W}$. The microstructure of the material strained to $\varepsilon_{\mathrm{HPT}}=14.3$ (not shown) reveals similar features.
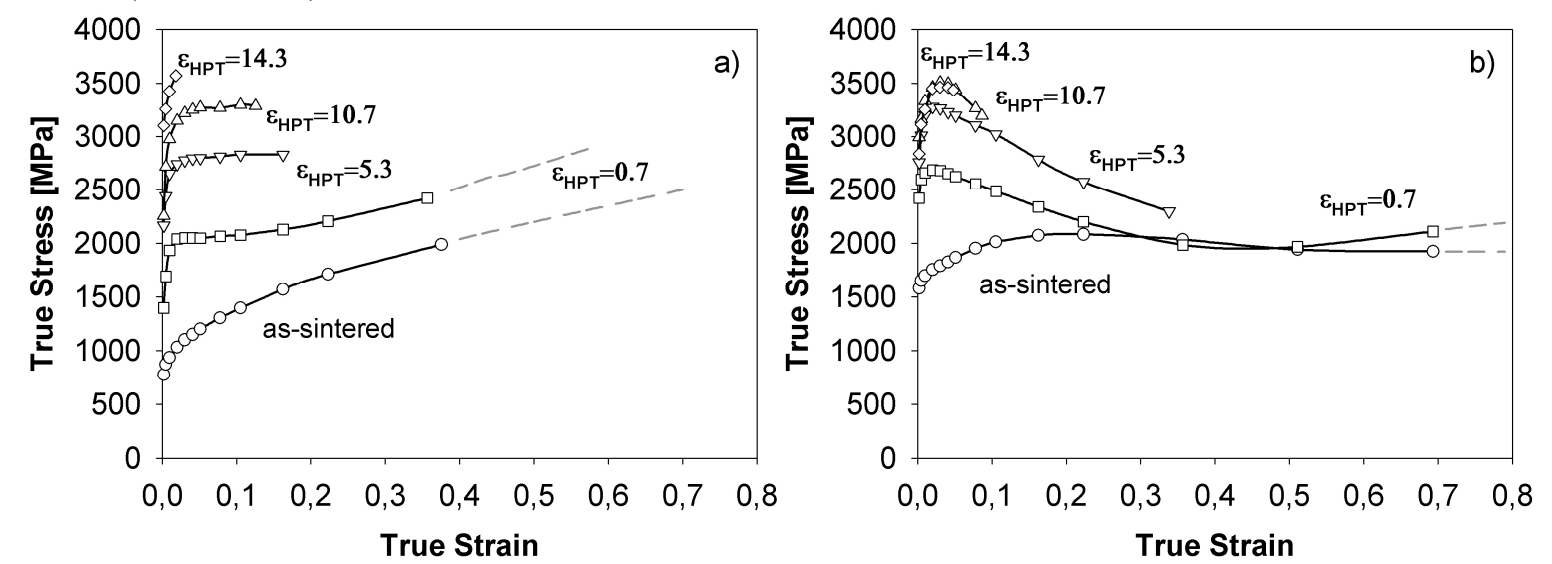

Fig. 3: Compressive stress-strain curves of the HPT-processed WHA under a) quasi-static and b) dynamic loading. Displayed strains are plastic strains.

In Figure 3 typical stress-strain curves for the RT HPT-processed WHA at quasi-static and dynamic compressive loading are shown. The as-sintered material shows strong strain hardening. After a rapid strength increase at the very beginning of plastic deformation below strains of $\sim 0.03$, the HPTprocessed conditions show insignificant strain hardening until shear fracture occurs or the contact friction increases. Due to the larger contacted surface area, the friction effect is more pronounced when compared to conventional sample geometries (height/diameter $\sim 1.6$ ). Nevertheless, the initial strain hardening might be attributed to both the strain gradient across the samples and the change in strain path from simple shear to compression. At dynamic loading the stress-strain curves change remarkably, showing flow-softening as the plastic work is converted into heat at quasi-adiabatic conditions.

For an illustration of the development of strength with increasing HPT pre-strain, the yield stresses (YS) for quasi-static and dynamic loading at 1\% plastic strain are plotted in Figure 4a. The largest increase in strength is achieved during the beginning of HPT.
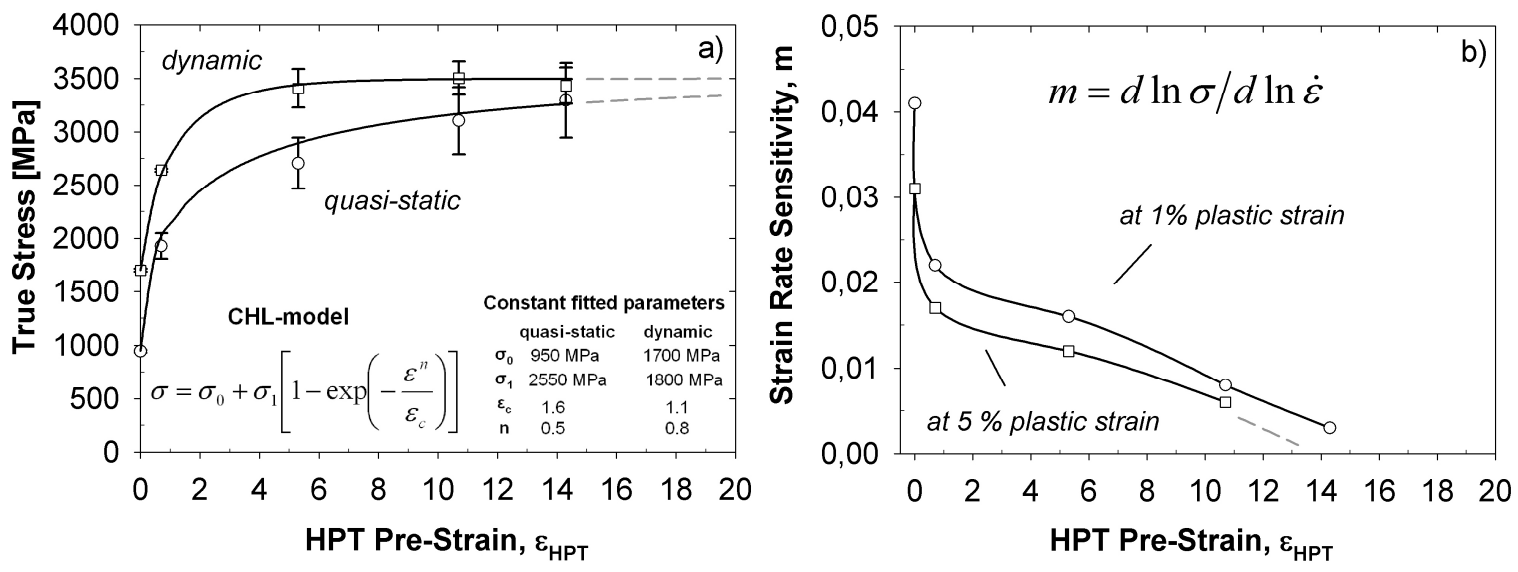

Fig. 4: Influence of HPT pre-strain on a) the strength (1\% yield stress) at quasi-static and dynamic loading and $b$ ) the strain rate sensitivity of the investigated WHA. 
At quasi-static loading after a strain of only 0.7 the YS is more than doubled to $1930 \mathrm{MPa}$. Further working of the material results in a lower increase in strength, since the formation and annihilation of defects is equalised. Chin, Horita and Langdon [15] showed that this behaviour can be described by an exponential power-law constitutive relationship, based on a theoretical examination of the evolution of dislocation densities. Accordingly, the YS approaches a maximum value of $\sim 3500$ $\mathrm{MPa}$. The YS at dynamic loading shows similar characteristics while the same limit is reached, but at much lower strain.

The influence of HPT on the strain rate sensitivity (SRS) is shown in Figure 4b. Unlike the strength it is strongly reduced during the beginning of plastic deformation. Further processing yields to a less pronounced decrease. It is well known that the mobility of screw dislocations is the rate controlling mechanism for bcc metals below temperatures below $\sim 30 \%$ of the melting temperature $[16,17]$. This is due to their thermally activated movement and interaction with short range obstacles such as forest dislocations which have to be overcome. This interaction is described by the so called PeierlsNabarro barrier. According to the comprehensive results in [18] the SRS of bcc metals decreases with a reduction of grain size, since new grain boundaries act as long-range obstacles that can not be overcome by thermal activation. For that reason, the SRS-plot implies that the interaction of moving dislocations with short range obstacles drops significantly already for considerably low strains and

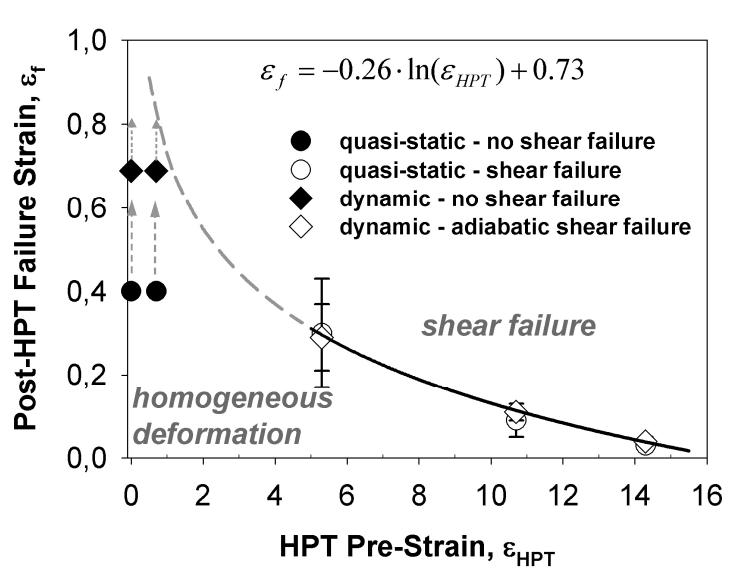
even disappears for strains of $\sim 15$. As a consequence, identical flow stresses for quasi-static and dynamic loading are reached. The ultimate stresses measured for both quasi-static and dynamic loading were $\sim 3600$ MPa for the material pre-deformed to a strain of 14.3 . This truly remarkable strength is $500 \mathrm{MPa}$ above the quasi-static value reported for pure $\mathrm{W}$ in [13]. The corresponding post-HPT-strain of only $\sim 3 \%$ is notably low.

Fig. 5: Post-HPT-strain for the investigated WHA

According to Figure 5 shear fracture occurred for quasi-static and dynamic loading for $\varepsilon_{\mathrm{HPT}}$ above 5.3, following a logarithmic trend. The samples strained to $\varepsilon_{\mathrm{HPT}}=0.7$ as well as the as-sintered materials did not show such behaviour for the strains applied in pure compression loading.

Thus, it can be concluded that the fracture for the quasi-static loading is most likely caused by stress singularities due to the constrained lateral straining, as rising contact friction exerted at the front faces of the samples. The dynamic loaded samples underwent adiabatic shearing as well. Figure 6

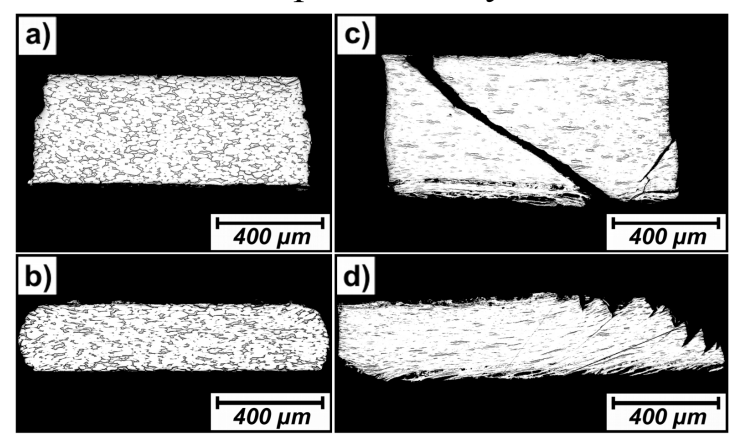
shows exemplary micrographs of recovered compression samples. The fracture always occurred by a major (adiabatic) shear band at an angle of $\sim 45^{\circ}$ with respect to the loading direction.

Fig. 6: Recovered compression samples for a) and c) quasi-static and b) and d) dynamic loading of a) and b) the as-sintered and c) and d) the $\varepsilon_{\mathrm{HPT}}=10.7$ condition

\section{Summary and Conclusions}

The microstructure of a tungsten heavy alloy $(92 \% \mathrm{~W}$, Ni-Co matrix) was refined by HPT at RT up to equivalent strains of 14.3 and investigated gradually. The HPT-strain was found to concentrate on the softer matrix, inducing a refinement down to a grain size of $\sim 80 \mathrm{~nm}$. In contrast the significant harder $\mathrm{W}$ remained partially refined, showing a substructure with a cell size of $\sim 250 \mathrm{~nm}$. Generally 
the largest change in mechanical properties is achieved by the application of considerably low HPTstrains, while during further HPT-processing the changes of the mechanical properties become less pronounced. Hence, the largest increase in strength is generated during the beginning of HPT, where after a strain of only 0.7 the YS is more than doubled to $\sim 1930 \mathrm{MPa}$. Further working of the material results in a lower increase in strength since the formation and annihilation of defects is equalised. A maximum stress of $\sim 3600 \mathrm{MPa}$ is reached for strains above $\sim 10$. Such remarkably strength being almost four times the yield stress of the as-sintered material has never been reported before for similar materials. Simultaneously, the strain hardening capacity is reduced significantly, resulting in fracture of the samples after considerably low post-HPT-strains. The first incidence of (adiabatic) shear banding appears after an HPT-strain of 5.3, indicated by an abrupt drop of flow stress and clear shear band formations. The strain rate sensitivity (SRS) is reduced drastically to half of the value by the application of a considerable low HPT-strain of 0.7 . For very high strains the SRS approaches zero, leading to identical flow stresses for both quasi-static and dynamic loading.

\section{Acknowledgements}

The authors gratefully acknowledge the Wehrwissenschaftliches Institut (WIWEB) for supporting this work and also thank the Deutsche Forschungsgemeinschaft (DFG) for partially supporting this work within the framework of Sonderforschungsbereich 692.

\section{References}

[1] S. J. Park, J. M. Martin, J. F. Guo, J. L. Johnson, R. M. German, Metallurgical and Materials Transactions A: Physical Metallurgy and Materials Science 37 (2006) 2837-2848.

[2] R. Z. Valiev, R. K. Islamgaliev, I. V. Alexandrov, Progress in Materials Science 45 (2000) 103189.

[3] R. Pippan, F. Wetscher, M. Hafok, A. Vorhauer, I. Sabirov, Advanced Engineering Materials 8 (2006) 1046-1056.

[4] S. Ferrasse, V. M. Segal, F. Alford, J. Kardokus, S. Strothers, Materials Science and Engineering: A In Press, Corrected Proof.

[5] R. Z. Valiev, T. G. Langdon, Progress in Materials Science 51 (2006) 881-981.

[6] Y. Ivanisenko, R. K. Wunderlich, R. Z. Valiev, H. J. Fecht, Scripta Materialia 49 (2003) 947952.

[7] A. Kostka, K. G. Tak, R. J. Hellmig, Y. Estrin, G. Eggeler, Acta Materialia 55 (2007) 539-550.

[8] F. Wetscher, R. Stock, R. Pippan, Materials Science and Engineering: A 445-446 (2007) 237243.

[9] I. Sabirov, R. Pippan, Scripta Materialia 52 (2005) 1293-1298.

[10] M. Faleschini, H. Kreuzer, D. Kiener, R. Pippan, Journal of Nuclear Materials 367-370 (2007) 800-805.

[11] A. Vorhauer, R. Pippan, Microstructure and thermal stability of tungsten based materials processed by means of severe plastic deformation, in: T. Chandra, J. M. Torralba, T. Sakai (Eds.), Materials Science Forum, vol 426-432, Madrid, 2003, pp. 2747-2752.

[12] Q. Wei, T. Jiao, K. T. Ramesh, E. Ma, L. J. Kecskes, L. Magness, R. Dowding, V. U. Kazykhanov, R. Z. Valiev, Acta Materialia 54 (2006) 77-87.

[13] Q. Wei, H. T. Zhang, B. E. Schuster, K. T. Ramesh, R. Z. Valiev, L. J. Kecskes, R. J. Dowding, L. Magness, K. Cho, Acta Materialia 54 (2006) 4079-4089.

[14] A. Vorhauer, R. Pippan, Scripta Materialia 51 (2004) 921-925.

[15] N. Q. Chinh, G. Horvath, Z. Horita, T. G. Langdon, Acta Materialia 52 (2004) 3555-3563.

[16] H. Conrad, Journal of Metals 16 (1964) 582-588.

[17] M. S. Duesbery, V. Vitek, Acta Materialia 46 (1998) 1481-1492.

[18] Q. Wei, S. Cheng, K. T. Ramesh, E. Ma, Materials Science and Engineering A 381 (2004) 7179. 